\title{
MEASURE THEORETIC GEOMETRY AND ELLIPTIC VARIATIONAL PROBLEMS ${ }^{1}$
}

\author{
BY F. J. ALMGREN, JR.
}

1. This article is intended as a survey of some of the phenomena and some of the recent results associated with higher dimensional boundary value problems in "parametric form" in the calculus of variations. These boundary value problems arise in the following way: Suppose $m$ and $n$ are positive integers and one is given a reasonably nice function $F: R^{m+n} \times \Gamma_{m} \rightarrow R^{+}$where $\Gamma_{m}$ denotes the Grassmann manifold of all unoriented $m$ plane directions in $R^{m+n}$ (which can be regarded as the space of all unoriented $m$ dimensional planes through the origin in $R^{m+n}$ ). If $S$ is a reasonably nice surface of dimension $m$ in $R^{m+n}$, one defines the integral $F(S)$ of $F$ over $S$ by setting

$$
F(S)=\int_{x \in S} F(x, S(x)) d H_{m} x
$$

where $S(x)$ denotes the tangent $m$ plane direction to $S$ at $x$ and $H_{m}$ denotes $m$ dimensional Hausdorff measure on $R^{m+n}$. Hausdorff $m$ dimensional measure gives a precise meaning to the notion of $m$ dimensional area in $R^{m+n}$ and is the basic measure used in defining a theory of integration over $m$ dimensional surfaces in $R^{m+n}$ which may have singularities. The Hausdorff $m$ dimensional measure of a smooth $m$ dimensional submanifold of $R^{m+n}$ agrees with any other reasonable definition of the $m$ area of such a manifold. With this terminology the problem can be stated:

Problem. Among all $m$ dimensional surfaces $S$ in $R^{m+n}$ having a prescribed boundary, is there one minimizing $F(S)$ ? And if there is, how nice is it?

To make this problem precise there are, of course, several questions to be answered:

(1) What is a surface?

(2) What is the boundary of a surface?

(3) What are reasonable conditions to put on $F$ ?

To see what is involved in answering these three questions, it is useful to consider some of the phenomena which arise. For these examples, we fix $F$ to be identically $1 . F(S)$ is thus the $m$ dimensional

\footnotetext{
1 An address delivered to the Society on April 12, 1968 by invitation of the Committee to Select Hour Speakers for Eastern Sectional Meetings; received by the editors September 26, 1968.
} 
area of $S$ and the problem is that of finding a surface of least $m$ dimensional area among all surfaces having a prescribed boundary. This problem is sometimes known as Plateau's problem (in honor of the Belgian physicist, J. Plateau, of the last century who, among other things, studied the geometry of soap films).

The first person to make significant progress on the study of Plateau's problem was the late J. Douglas. He showed, in particular, the following result $[D]$ : If $C$ is a simple closed rectifiable curve in $R^{3}$ and $D_{0}$ is the two dimensional disk, then among all continuous mappings $D_{0} \rightarrow R^{3}$ which map $\partial D_{0}$ homeomorphically onto $C$ and are smooth on the interior of $D_{0}$ there is a mapping $f$ of least area, where the area of $f$ means

$$
\int_{D_{0}}\left|f_{\sharp}(\partial / \partial u \wedge \partial / \partial v)\right| d H_{2}
$$

when $u$ and $v$ are orthonormal coordinates for $D_{0}$ or, equivalently, the Hausdorff 2 dimensional measure of $f\left(D_{0}\right)$ counting multiplicities, i.e.

$$
\int_{x \in f\left(D_{0}\right)} N(f, x) d H_{2} x
$$

where $N(f, x)$ is the number (possibly $\infty$ ) of points in $f^{-1}(x)$. The equivalence of these two integrals for the area of $f$ indicates one of the reasons it is sometimes useful to consider surfaces with multiplicities. (The multiplicity of the integral varifold $f_{f}\left|D_{0}\right|$ equals $N(f, x)$ for $\boldsymbol{H}_{2}$ almost all $x$.) There are no known direct generalizations of this result of Douglas to higher dimensional disks and higher dimensional areas, and the methods of Douglas are strictly 2 dimensional.

Suppose again that $C$ is a simple closed rectifiable curve in $R^{3}$ and $D_{m}$ denotes the 2 dimensional disk with $m$ handles for $m=0,1,2, \ldots$. (see Figure 1).

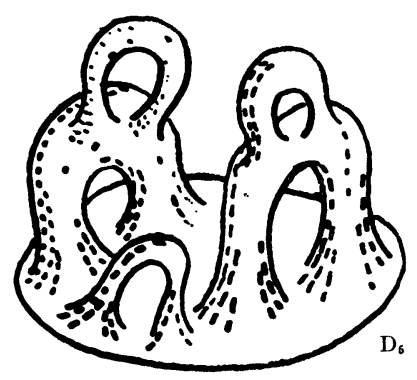

FIGURE 1. 
Let $A_{m}$ denote the infimum of the areas of the continuous mappings $D_{m} \rightarrow R^{3}$ which map $\partial D_{m}$ homeomorphically onto $C$ and which are smooth on the interior of $D_{m}$. In general, mappings realizing this infimum value are not known to exist. Since one can always "pinch out" a handle before mapping $D_{m}$ into $R^{3}$, one has $A_{0} \geqq A_{1} \geqq A_{2} \geqq \cdots$ $\geqq \lim _{m} A_{m} \geqq 0$. W. H. Fleming [FL 1] has shown, however, that if the curve $C$ resembles that shown in Figure 2, then one has the strict inequalities $A_{0}>A_{1}>A_{2}>\cdots>\lim _{m} A_{m}>0$.

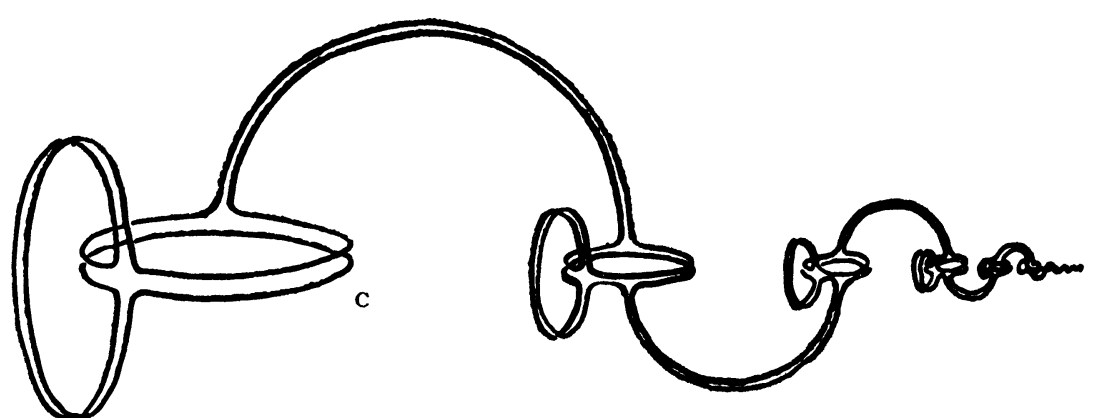

FIGURe 2.

This curve $C$ is a simple closed unknotted curve of finite length which is smoothly imbedded except at one point. If one wishes to find an oriented surface of absolute least area among such surfaces having $C$ as boundary, i.e. whose area equals $\lim _{m} A_{m}$, then there does not exist such a surface having finite topological type since the list of $A_{m}$ 's contains every compact orientable 2 dimensional manifold having a circle as boundary. On the other hand there does exist a surface, sketched in Figure 3, which deserves to be called the oriented surface of least area having $C$ as boundary. Its area is $\lim _{m} A_{m}$ and this sur-

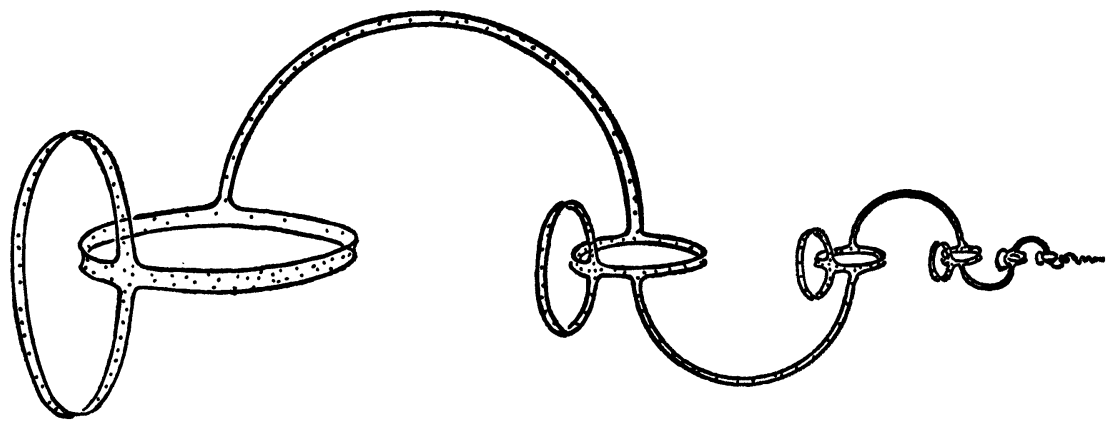

FIGURE 3. 
face at all interior points is a 2 dimensional real analytic manifold having 0 mean curvature. Topologically this surface is a disk with a countable number of handles converging to a boundary point. This example illustrates why in order to solve the least area problem, and really achieve the least area, one sometimes has to admit surfaces of infinite topological type into competition.

The following example due to $\mathrm{H}$. Federer $[\mathrm{F4}, 4]$. Let $V$ denote a complex algebraic variety of complex dimension $k$ in complex $n$ dimensional space $\boldsymbol{C}^{n}$, and regard $V$ as a real $2 k$ dimensional surfare oriented by its complex structure. Now let $V_{0}$ denote a bounded relatively open subset of $V$ having a well-defined oriented boundary of finite $2 k-1$ dimensional measure, and let $W$ be another oriented surface with $\partial W=\partial V_{0}$. Then the $2 k$ dimensional area of $W$ is at least as large as the $2 k$ dimensional area of $V_{0}$, and this is true whether or not $V_{0}$ contains singularities. In particular, $V_{0}$ is a solution to Plateau's problem for oriented $2 k$ dimensional surfaces having $\partial V_{0}$ as boundary provided one is willing to admit complex algebraic varieties as solutions. If one does admit complex algebraic varieties as solutions, then one must accept as singularities in solutions to Plateau's problem at least all the singularities which occur as singularities in complex algebraic varieties.

There are other reasons which force surfaces of least area to have singularities. R. Thom [T, III.9] has given an example of a compact, real analytic, 14 dimensional manifold $M$ having a 7 dimensional integral homology class $\sigma$ which cannot be represented by a smoothly imbedded, or even a smoothly immersed, differentiable manifold of dimension 7. On the other hand every integral homology class in every compact Riemannian manifold can be represented by an oriented surface (more precisely a minimal integral current) of least area among all surfaces representing that class [FF, 9.6]; in particular there is a 7 dimensional surface $S$ in $M$ representing $\sigma$ which has area no larger than that of any other surface representing $\sigma . S$ must contain singular points; otherwise it would be a 7 dimensional real analytic submanifold of $M$ and as such contradict the result of Thom.

To see what is involved in the question of boundaries, it is useful to ask what solution one would like for certain least area problems for simple closed curves. There is one case about which everyone agrees: if $C_{3}$ is a standard circle then the unique surface of least area having $C_{1}$ as boundary should be the flat disk $S_{1}$ (Figure 4).

Suppose $C_{2}$ is a curve which lies close to a circle but which goes around twice before it rejoins. One strong candidate for the surface 
1969]

MEASURE THEORETIC GEOMETRY

289
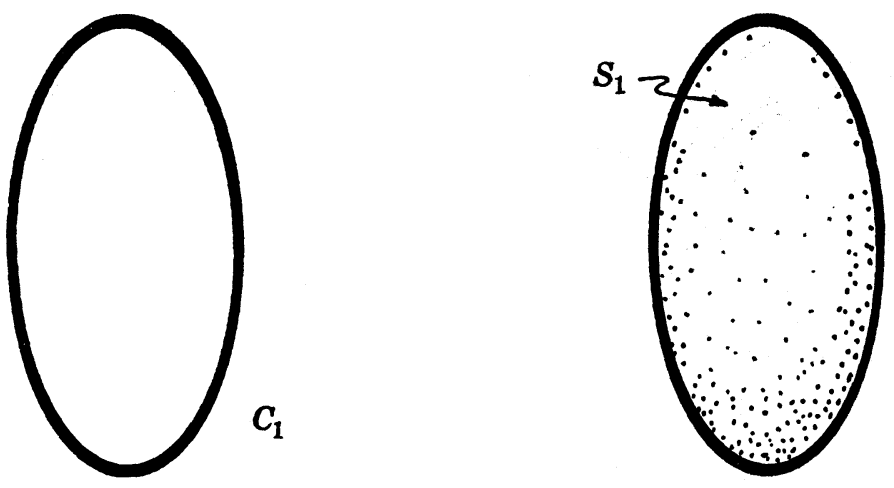

Figure 4.

of least area having $C_{2}$ as boundary is a Möbius band $S_{2}$ (see Figure 5).

Such a surface exists as a soap film on a boundary wire bent in the shape of $C_{2}$, and it also exists as a mathematical minimal surface of absolute least area in competition with all surfaces having $C_{2}$ as boundary in the sense of homology with coefficients in the integers modulo 2. Now suppose $C_{3}$ is a curve which lies close to a circle but which goes around three times before it rejoins itself. It is useful to think of such a curve as lying on a torus and being swept out as three equally spaced points on a meridian circle travel around the torus the long way while the meridian circle makes one third of a revolution (see Figure 6).

One strong candidate for the surface of least area having boundary $C_{3}$ is a triple Möbius band $S_{3}$ (Figure 6 ) which is swept out by a $Y$ shaped piece of curve between the three equally spaced points on a

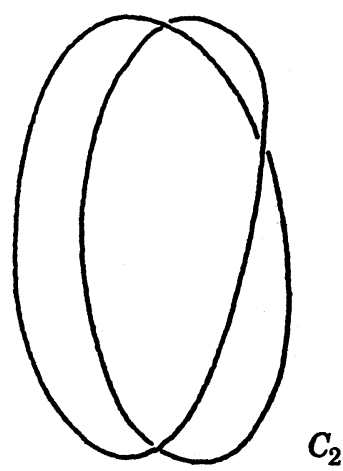

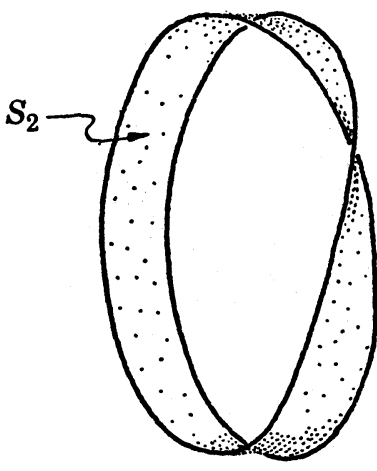

Figure 5. 
290

F. J. ALMGREN, JR.

[March
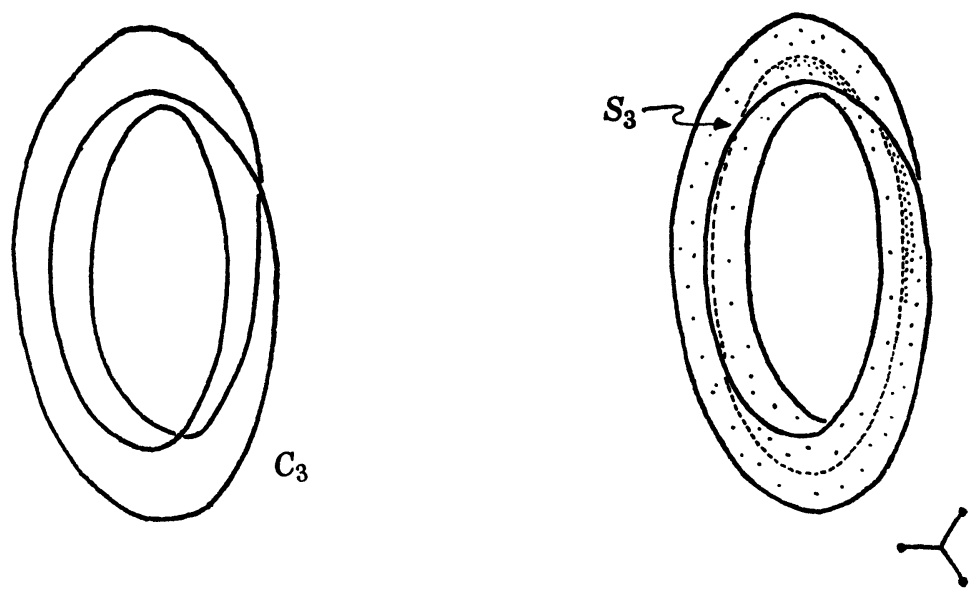

FIGURE 6.

meridian circle as this circle travels around the torus. Such a surface as $S_{3}$ exists as a soap film on a boundary wire bent in the shape of $C_{3}$, and it also exists as a mathematical minimal surface of absolute least area in competition with all surfaces having $C_{8}$ as boundary in the sense of homology with coefficients in the integers modulo 3 .

Suppose now one forms a new simple closed curve $C_{4}$ which looks like $C_{2}$ on the left attached by a thin bridge to $C_{3}$ on the right (Figure 7).

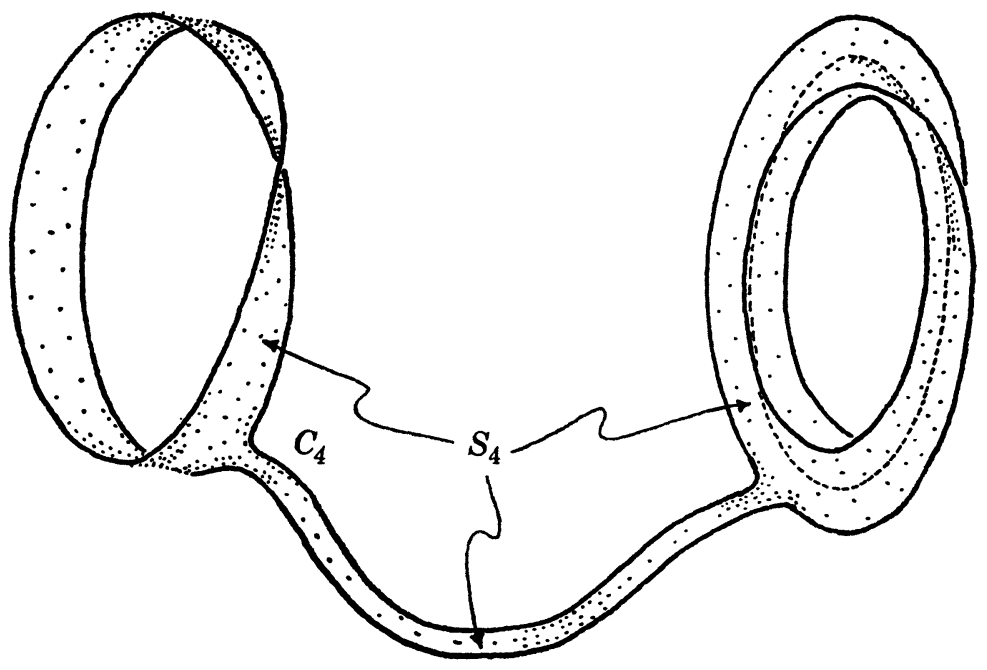

Figure 7. 
One probably should want the surface $S_{4}$ of least area having $C_{4}$ as boundary to look like $S_{2}$ on the left attached to $S_{3}$ on the right by a thin ribbon of surface. Such a surface $S_{4}$ exists as a soap film on a boundary wire in the shape of $C_{4}$ and also exists as a mathematical minimal surface. On the other hand, F. Adams has constructed a continuous map $\left(S_{4}, C_{4}\right) \rightarrow\left(C_{4}, C_{4}\right)$ retracting $S_{4}$ onto $C_{4}$ [R1, p. 80]. Thus in no way in the sense of algebraic topology does the surface $S_{4}$ have the curve $C_{4}$ as its boundary. In particular, in order to study surfaces of the type of $S_{4}$ as solutions to variational problems, one needs alternative notions of boundary to those of algebraic topology.

There is another phenomenon which should be mentioned at this point. If one bends a wire in the shape indicated in Figure 8-1 and dips it in a soap solution, one of the soap films which can form is sketched also in Figure 8-2. Note the singular curve where the two sheets of surface "pass through each other." If one punctures the lower region, surprisingly the entire film is not destroyed, but rather assumes the shape sketched in Figure 8-3. Here then is a "minimal surface" which does not touch all of its boundary, and one can even cut off the extra boundary without destroying the film. A mathematical minimal surface like that of 8-3 does exist, but only on a wire having positive thickness, i.e. no such surface can exist on an infinitely thin boundary.

2. Surfaces as measures and surfaces as maps. One approach to studying variational problems in the generality suggested by the preceding examples is based on a correspondence between suitable surfaces and measures on appropriate spaces. Indeed, the natural setting for parametric ${ }^{2}$ problems in the calculus of variations seems to be that in which surfaces are regarded as intrinsically part of $R^{m+n}$ (in particular as measures on spaces associated with $R^{m+n}$ ) rather than that in which surfaces are regarded as mappings from a fixed $m$ dimensional manifold, even though this approach necessitates giving up most of the traditional methods of functional analysis for showing the existence of solutions. The following are the main reasons for formulating the problem in this way.

\footnotetext{
${ }^{2}$ Traditionally one has considered surfaces as mappings $f$ from a fixed $m$ dimensional manifold $M$ into $R^{m+n}$ and attempted to minimize the integral of a suitable integrand. If the integrand depends only on $x \in M, f(x)$, and $D f(x)$ and if the integral of the integrand is independent of the parametrization of $M$ (as is the case, for example, for the area integrand, but is not the case for most "energy" integrands), the variational problem said to be in parametric form [M1, 9.1]. Problems in parametric form are precisely those problems for which the necessary integration can be performed over the image $f(M)$ in $R^{m+n}$.
} 


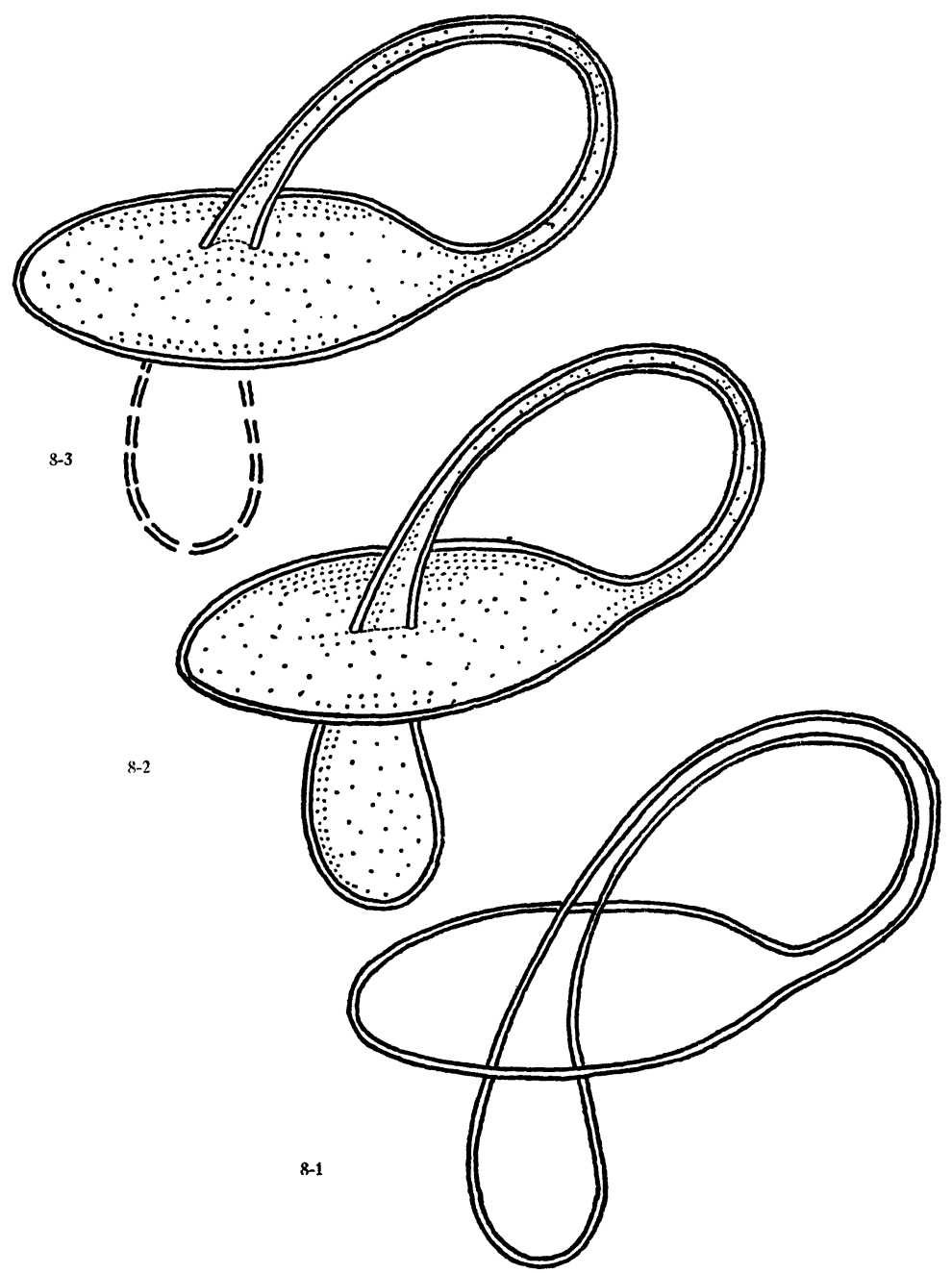

FIGURE 8.

(1) If one regards surfaces as mappings from a fixed compact $m$ dimensional manifold, then the resulting theory cannot take into account the phenomena of the examples. Indeed one cannot consider surfaces of infinite topological type, or surfaces having singularities not realizable by mappings (like those of the surface $S_{3}$ of Figure 6), or surfaces having boundaries defined in more sophisticated ways.

(2) Many significant results have been obtained from the study of parametric problems in the measure theoretic setting [A2], [A4], 
[A5], [DG1], [F4], [F5], [FF], [FL2], [FL3], [M1, 10], [R1], [R2], [R3] in contrast with the virtual absence of such results in higher dimensions and codimensions in the mapping setting.

(3) It seems reasonable to hope that once the singularities of measure theoretic solutions are understood, one will be able to solve the mapping problem as a consequence.

(4) The topological methods of the Morse theory are available in the measure theoretic setting [A2] in contrast, with the absence of such methods in the mapping setting, i.e. the "Condition (C)" of R. Palais and S. Smale [PS] is not satisfied by any variational problem in parametric form.

(5) The techniques developed in the study of variational problems in the measure theoretic setting have aided in the solution of related problems; for example:

(a) Proof of regularity almost everywhere of weak solutions to some nonlinear elliptic systems of partial differential equations [M2], [GM].

(b) Generalizations of Bernstein's theorem in dimensions up to 8 [A4], [DG2], [FL2], [S].

(c) Various long-standing questions in the theory of Lebesgue area have been settled [F3].

(d) The basic theorems of integral geometry have been proved in their most natural generality [B].

(6) In the measure theoretic setting, many important geometric constructions are available whose analogues in spaces of mappings appear unnatural $[\mathrm{A1}],[\mathrm{A2}],[\mathrm{A} 5],[\mathrm{FF}],[\mathrm{FL} 2],[\mathrm{FL} 3],[\mathrm{R} 1],[\mathrm{R2}]$.

3. Geometric measure theory. The creation of a natural and powerful theory of measure and integration over $m$ dimensional surfaces in $R^{m+n}$ which may contain nontrivial singularities has, more than anything else, been the object of those results in mathematics collectively called "geometric measure theory." It is now understood, for example, in what natural generality Stokes' Theorem is true [F4, 2.2]. A definitive work, Geometric Measure Theory, covering both general measure theory and geometric measure theory, will be published by $\mathrm{H}$. Federer within the next year. Among those who have made major contributions to distinctly geometric measure theory are A. S. Besicovitch, A. P. Morse, H. Federer, W. H. Fleming, E. De Giorgi, and E. R. Reifenberg. Also H. Whitney's definition of the "flat norm" has been crucial in some applications, and L. C. Young's "generalized surfaces" were a predecessor to normal and integral currents. 
The following definitions are essential in discussing geometric measure theory.

(a) Hausdorff measure. For each $k, 0 \leqq k \leqq m+n, H_{k}$ denotes Hausdorff $k$ dimensional measure in $R^{m+n}$. For $A \subset R^{m+n}, H_{k}(A)$ equals the limit as $r \rightarrow 0^{+}$of the infimum of the sums $\sum_{B \in F} 2^{-k} \alpha(k)$ diameter $(B)^{k}$ corresponding to all countable coverings $F$ of $A$ such that diameter $(B)<r$ for $B \in F$. Here $\alpha(k)$ denotes the $k$ area of the unit $k$ ball. $H_{k}$ is a Borel regular measure with $H_{m+n}$ equal to Lebesgue measure $L_{m+n}$.

(b) Rectifiable sets. A set $S \subset R^{m+n}$ is called $k$ rectifiable if and only if $S$ is bounded, $H_{k}$ measurable, and for each $\epsilon>0$ there exists a compact $k$ dimensional submanifold $M$ of $R^{m+n}$ of class 1 such that $H_{k}([S \sim M] \cup[M \sim S])<\epsilon$. If $S$ is $k$ rectifiable then for $H_{k}$ almost all $x \in S, S$ has an approximate tangent plane $S(x) \in \Gamma_{k}$. The mapping $S: S \rightarrow \Gamma_{k}$ sending $H_{k}$ almost all $x \in S$ to $S(x)$ is $H_{k}$ measurable. By an oriented $k$ rectifiable set $S$, one means a $k$ rectifiable set $S$ together with an $H_{k}$ measurable map $S^{*}: S \rightarrow \Gamma_{k}^{*}$ of $S$ into the Grassmann manifold $\Gamma_{k}^{*}$ of oriented $k$ plane directions in $R^{m+n}$ with $g \circ S^{*}=S H_{k}$ almost everywhere, where $g: \Gamma_{k}^{*} \rightarrow \Gamma_{k}$ is the natural covering.

(c) Purely unrectifiable sets. A set $U \subset R^{m+n}$ is called purely $k u n$ rectifiable if and only if $U$ is bounded and $H_{k}$ measurable and $U$ contains no $k$ rectifiable subset of positive $H_{k}$ measure.

The key role of Hausdorff measure, rectifiable sets, and purely unrectifiable sets is illustrated in the following theorem (due in general dimensions to $H$. Federer $[F 1,9.6])$.

Structure Theorem for Sets of Finite Hausdorff Measure. (1) Let $A \subset R^{m+n}$ be bounded and $H_{k}$ measurable with $H_{k}(A)<\infty$. Then $A=R \cup U H_{k}$ almost uniquely where $R$ is $k$ rectifiable, $U$ is purely $k$ unrectifiable, and $R \cap U=\varnothing$.

(2) If $U \subset R^{m+n}$ is purely $k$ unrectifiable, then $H_{k}(\pi(U))=0$ for almost all orthogonal projections $\pi: R^{m+n} \rightarrow R^{k}$ in the usual orthogonally invariant measure on the space of such projections.

4. Measure theoretic surfaces. The most commonly used measure theoretic surfaces are the following:

(a) Variational measures. If $S \subset R^{m+n}$ is $m$ rectifiable, the variation measure $\|S\|$ associated with $S$ is given by the formula $\|S\|=H_{m} \cap S$ (i.e. $\|S\|(A)=H_{m}(S \cap A)$ for $\left.A \subset R^{n}\right)$. $\|S\|$, of course, determines $S H_{m}$ almost uniquely but it is difficult to evaluate $F(S)$ from $\|S\|$ alone.

(b) Integral varifolds. If $S \subset R^{m+n}$ is $m$ rectifiable, the integral vari- 
fold $|S|$ is given by the formula

$$
|S|=\phi_{f}\left(H_{m} \cap S\right)=\phi *(\|S\|),
$$

where $\phi: R^{m+n} \rightarrow R^{m+n} \times \Gamma_{m}, \phi(x)=(x, S(x))$ for $\|S\|$ almost all $x$ in $R^{m+n}$, i.e.

$$
|S|(B)=H_{m}(S \cap\{x:(x, S(x)) \in B\}) \quad \text { for } B \subset R^{m+n} \times \Gamma_{m} .
$$

Note that $F(S)=\int F d|S|$. A measure $V$ on $R^{m+n} \times \Gamma_{m}$ is called an integral varifold of dimension $m$ if and only if there exists a uniformly bounded family $S_{1}, S_{2}, S_{3}, \ldots$ of $m$ rectifiable sets such that $V$ $=\sum_{i}\left|S_{i}\right|$ and $M(V)=\sum_{i} H_{m}\left(S_{i}\right)<\infty . V_{m}\left(Z^{+}\right)$denotes the family of all $m$ dimensional integral varifolds in $R^{m+n}$. Lipschitzian maps $f$ of Euclidean spaces induce maps $f_{\star}$ of $V_{m}\left(Z^{+}\right)$in a natural way. For variational problems in the varifold setting, the following extension of the notion of mean curvature is often important. For $V \in V_{m}\left(Z^{+}\right)$ and $W \in V_{m-1}\left(Z^{+}\right)$one sets

$$
\odot(V, W)=\sup \frac{\left.(d / d t) \boldsymbol{M}(v(t, \cdot) \nRightarrow V)\right|_{t=0}-\left.(d / d t) \boldsymbol{M}\left(v_{\sharp}([0, t] \times W)\right)\right|_{t=0}}{\left.(d / d t) \boldsymbol{M}\left(v_{f}([0, t] \times V)\right)\right|_{t=0}}
$$

where the supremum is taken over all class 3 deformations $v: R \times R^{m+n}$ $\rightarrow R^{m+n}$ with $v(0, x)=x, x \in R^{m+n}$, and $\left.(d / d t) M(v+([0, t] \times V))\right|_{t=0}>0$. If $M$ is a compact class 2 manifold of dimension $m$, then $\odot(|M|,|\partial M|)$ equals $m$ times the maximum mean curvature of $M$ in $R^{m+n}$, this maximum being taken over all points and all normal directions at each point. If $M$ is a minimal submanifold, then, of course, $\mathcal{P}(|M|,|\partial M|)=0$. Suppose $W \in V_{m-1}\left(Z^{+}\right)$and $V \in V_{m}\left(Z^{+}\right)$. One calls $V$ stationary for the boundary $W$ if and only if $P(V, W)=0$ and regular for the boundary $W$ if and only if $\rho(V, W)<\infty$. Stationary integral varifolds seem to include mathematical models for all soap films while regular integral varifolds seem to include models for all soap bubbles (see Figure 9). Among the most important properties of the spaces of surfaces commonly used in geometric measure theory are their compactness properties. For integral varifolds one has

CompactNess Theorem [A2, 10.8]. Let $K \subset R^{m+n}$ be compact and $M<\infty$. Then

$$
\begin{aligned}
V_{m}\left(Z^{+}\right) \times V_{m-1}\left(Z^{+}\right) & \cap(V, W): \operatorname{spt}(V) \subset K, \operatorname{spt}(W) \subset K, \\
& M(V) \leqq M, M(W) \leqq M, \odot(V, W) \leqq M, \odot(W, 0) \leqq M\}
\end{aligned}
$$

is compact in the weak topology. 


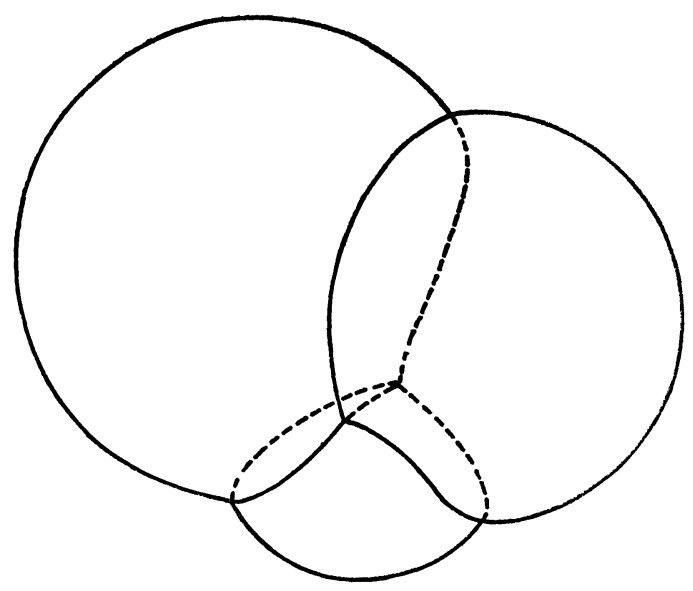

Figure 9. A regular integral varifold.

One consequence of topological arguments similar to those of the Morse Theory is the following existence theorem for (generally unstable) stationary integral varifolds.

TheOREM [A2, 15.2]. Let $M \subset R^{m+n}$ be a compact $n$ dimensional Riemannian manifold of class 3 without boundary. Then for each $k, 0 \leqq k \leqq n$, there exists a $k$ dimensional integral varifold lying on $M$ which is stationary (for boundary 0 ) under every variation of $R^{m+n}$ carrying $M$ into itself.

The following results give various estimates in the large which depend only on bounds for $P$.

ISOPERIMETRIC INEQUALITY [A2, 8.7]. There exist (easily computed) constants $a>0$ and $b<\infty$ such that if $m \geqq 2, V \in V_{m}\left(Z^{+}\right), W \in V_{m-1}\left(Z^{+}\right)$ with

$$
\odot(V, W)^{m} M(V) \leqq a,
$$

then

$$
M(V) \leqq b M(W)^{m /(m-1)} .
$$

THEOREM. There exists an easily computed constant $C>0$ such that if

$$
\begin{gathered}
V \in V_{m}\left(Z^{+}\right), \quad W \in V_{m-1}\left(Z^{+}\right), \quad \rho(V, W)=0, \\
\operatorname{dist}(0, \operatorname{spt}(W)) \geqq R>0, \quad \operatorname{dist}(0, \operatorname{spt}(V)) \leqq R-\epsilon>0,
\end{gathered}
$$

then $M(V) \geqq C(R \epsilon)^{m / 2}$. 
THEOREM [A2, 8.2(3)]. Let $V \in V_{m}\left(Z^{+}\right), W \in V_{m-1}\left(Z^{+}\right), \rho(V, W)$ $=0$. Then $r^{-m} M(V \cap\{x:|x|<r\})$ is monotonically nondecreasing as a function of $r$ for $0<r<\operatorname{dist}(0, \operatorname{spt}(W))$.

THEOREM [A2, 9.4]. There exists $2<L<\infty$ with the following property. Suppose: $V \in V_{m}\left(Z^{+}\right), W \in V_{m-1}\left(Z^{+}\right)$with $\rho(V, W)=0$. If $\operatorname{spt}(W) \subset A=\{x:|x| \leqq 1$ or $|x-v| \leqq 1\}$, for some $v \in R^{m+n}$ with $|v| \geqq L$, then $\operatorname{spt}(V) \subset A$.

(c) Integral currents. If $S$ is an oriented $m$ rectifiable subset of $R^{m+n}$, the current associated with $S$ is the continuous linear function $S: E^{m} \rightarrow R$ on the space $E^{m}$ of $C^{\infty} m$ forms on $R^{m+n}$ given by $S(\phi)=\int_{S} \phi$ for each $\phi \in E^{m}$. $S$ can also be regarded as an $m$ vector valued measure on $R^{m+n}$ in which case, $S(\phi)=\int \phi d S$. A current $Q: E^{m} \rightarrow R$ (i.e. continuous linear functional) is called an integral current (or $Z$ chain) if and only if there exist a uniformly bounded disjointed family $S_{1}, S_{2}$, $S_{3}, \cdots$ of oriented $m$ rectifiable sets and a uniformly bounded disjointed family $T_{1}, T_{2}, T_{3}, \ldots$ of oriented $m-1$ rectifiable sets such that $Q=\sum_{i} i S_{i}$ and $\partial Q=\sum_{i} i T_{i}$ which means for each $\phi \in E^{m}, Q(\phi)$ $=\sum_{i} i \int \phi d S_{i}$, and for each $\Psi \in E^{m-1}, \partial Q(\Psi)=\sum_{i} i \int \Psi d T_{i}=Q(d \Psi)$, and such that $M(Q)=\sum_{i} \imath H_{m}\left(S_{i}\right)<\infty$ and $M(\partial Q)=\sum_{i} i H_{m-1}\left(T_{i}\right)$ $<\infty . \boldsymbol{Z}_{m}(Z)$ denotes the space of all $m$ dimensional integral currents in $R^{m+n} . Z_{m}(Z)$ has its usual weak topology and also the topology of the flat norm $L: \boldsymbol{Z}_{m}(Z) \rightarrow R^{+}$given by $L(Q)=\inf \{M(Q-\partial R)+M(R)$ : $\left.R \in Z_{m+1}(Z)\right\}$. These topologies agree on suitably bounded subsets of $\boldsymbol{Z}_{m}(Z)$, and $\boldsymbol{M}$ is lower semicontinuous in these topologies. The following results indicate some of the desirable properties of integral currents.

CompactNess Theorem [FF, 8.13]. Let $K \subset R^{m+n}$ be compact and $M<\infty$. Then $Z_{m}(Z) \cap\{Q: \operatorname{spt}(Q) \subset K, M(Q) \leqq M, M(\partial Q) \leqq M\}$ is compact in both the weak and the $L$ topologies (and for elliptic integrands $F$, the function sending $Q \in Z_{m}(Z)$ to $\int_{Q} F$ is lower semicontinuous).

THEOREM [FF, 5.11]. If $B C A \subset R^{m+n}$ are Lipschitz neighborhood retracts (for example differentiable submanifolds) then the homology groups of the chain complex

$$
\underset{k=0}{\oplus+n} Z_{k}(Z) \cap\{Q: \operatorname{spt}(Q) \subset A, \operatorname{spt}(\partial Q) \subset B\}
$$

are naturally isomorphic with the singular homology groups of $(A, B)$ with coefficients in $Z$. As one consequence, each $m$ dimensional homology 
class of $(A, B)$ can be represented by an $m$ dimensional integral current of least mass in that class.

THEOREM [A1, 7.1]. If $B \subset A$ are compact Lipschitz neighborhood retracts in $R^{m+n}$ then there is a natural isomorphism

$$
\pi_{i}\left(Z_{m}(A, B ; Z) ; 0\right) \cong H_{i+m}(A, B ; Z)
$$

between the ith homotopy group of the space $\boldsymbol{Z}_{m}(A, B ; Z)=\boldsymbol{Z}_{m}(Z)$ $\cap\{Q: \operatorname{spt}(Q) \subset A, \operatorname{spt}(\partial Q) \subset B\}$ and the $(i+m)$ th singular homology group of $(A, B)$ with coefficients in $Z$. This fact, in particular, is precisely the topological information relevant to Morse theory methods for finding higher dimensional minimal surfaces.

(d) $G$ chains. Suppose $G$ is a finitely generated abelian group with a translation invariant norm | |. By a $G$ chain $Q$, one means a surface having multiplicities in $G$ and having a well-defined boundary. More precisely $Q$ is a $G$ chain of dimension $m$ if and only if there exist a uniformly bounded disjointed family $\left\{S_{g}: g \in G \sim\{0\}\right\}$ of oriented $m$ rectifiable subsets of $R^{m+n}$ and a uniformly bounded disjointed family $\left\{T_{g}: g \in G \sim\{0\}\right\}$ of $m-1$ rectifiable subsets of $R^{m+n}$ such that

$$
Q=\sum_{g \in G \sim\{0\}} g S_{o} \text { and } \partial Q=\sum_{g \in G \sim\{0\}} g T_{o},
$$

and $\boldsymbol{M}(Q)=\sum_{\sigma}|g| H_{m}\left(S_{\vartheta}\right)<\infty$ and $\boldsymbol{M}(\partial Q)=\sum_{g}|g| H_{m-1}\left(T_{o}\right)<\infty$.

Here $\partial Q$ is defined in the sense of homology theory. One denotes by $\boldsymbol{Z}_{m}(G)$ the space of all $G$ chains of dimension $m$ in $R^{m+n}$. $\boldsymbol{Z}_{m}(G)$ usually carries the topology of the flat norm $L: Z_{m}(G) \rightarrow R^{+}$given by

$$
L(Q)=\inf \left\{\boldsymbol{M}(Q-\partial R)+\boldsymbol{M}(R): R \in \boldsymbol{Z}_{m+1}(G)\right\} .
$$

The three theorems for $Z$ chains in the preceding section remain true with $G$ replacing $Z$ [FL, 3].

5. One formulation of the problem. As the variety of different measure theoretic surfaces suggests, there are a number of different ways in which the problem of finding an $F$ minimal surface having a prescribed boundary can be formulated. The following formulation is an especially fundamental one.

Definitions. (a) A surface $S$ is a compact $m$ rectifiable subset of $R^{m+n}$.

(b) A boundary $B$ is a compact $m-1$ rectifiable subset $R^{m+n}$.

(c) $H_{m-1}(B ; G)$ denotes the $m-1$ dimensional Vietoris homology group of $B$ with coefficients in a finitely generated abelian group $G$. 
For $\sigma \in H_{m-1}(B ; G)$ (intuitively $\sigma$ is a hole in $B$ ) we say that $S$ spans $\sigma$ if and only if $i_{*}(\sigma)=0$ where

$$
i_{*}: \quad H_{m-1}(B ; G) \rightarrow H_{m-1}(B \cup S ; G)
$$

is induced by the inclusion $i: B \rightarrow B \cup S$.

(d) An integrand $F: R^{m+n} \times \Gamma_{m} \rightarrow R^{+}$is called elliptic with respect to $G$ if and only if there is a continuous positive function $c: R^{m+n} \rightarrow R^{+}$ such that for each $x \in R^{m+n}$, each $m$ disk $D$ in $R^{m+n}$, and each surface $S$ which spans some $\sigma \in H_{m-1}(\partial D ; G) \sim\{0\}$,

$$
F^{x}(S)-F^{x}(D) \geqq c(x)\left[H_{m}(S)-H_{m}(D)\right]
$$

where $F^{x}$ is the integrand given for $y \in R^{m+n}, \pi \in \Gamma_{m}$ by the formula, $F^{x}(y, \pi)=F(x, \pi)$. If the codimension $n$ equals 1 , the ellipticity of $F$ with respect to any $G$ is equivalent to the uniform convexity of each $F^{x}$. The set of elliptic integrands contains a (computable) convex neighborhood of the $m$ area integrand $F \equiv 1$ in the $C^{(2)}$ topology. Also if $R^{m+n} \rightarrow R^{m+n}$ is a diffeomorphism then $f_{\sharp} F$ is elliptic if and only if $F$ is. This fact extends the results of the following theorem from $R^{m+n}$ to compact $m+n$ dimensional Riemannian manifolds without boundary of class $j+1$. Finally the ellipticity of $F$ implies that the various Euler equations which arise are elliptic systems of partial differential equations, and "in the small" the ellipticity of $F$ is equivalent to the ellipticity of these systems.

With this terminology one can state the following theorem:

\section{TheOREM $[\mathbf{A 5}, 1.4]$.}

Hypotheses. (1) Let $B$ be a boundary.

(2) Let $G$ be a finitely generated abelian group.

(3) Let $\sigma \in H_{m-1}(B, G)$.

(4) Let $F: R^{m+n} \times \Gamma_{m} \rightarrow R^{+}$be an integrand of class $j \geqq 3$ which is elliptic with respect to $G$ and which is bounded away from 0 .

CoNCLUSION. There exists a surface $S$ with the following properties:

(1) $S$ spans $\sigma$.

(2) $F(S) \leqq F(T)$ whenever $T$ is a surface which spans $\sigma$.

(3) Except possibly for a compact singular set of zero $H_{m}$ measure, $S$ is an $m$ dimensional submanifold of $R^{m+n}$ of class $j-1$.

6. Construction of elliptic partial differential equations having discontinuous solutions. The invariance of the family of elliptic integrands $F: R^{m+n} \times \Gamma_{m} \rightarrow R^{+}$under diffeomorphisms of $R^{m+n}$ and the fact that the Euler equations associated with these integrands are elliptic systems of partial differential equations give an easy way 
of making partial differential equations whose solutions have various singularities, including discontinuities. For example, let $F: R^{3} \times \Gamma_{2}$ $\rightarrow\{1\}$ be the 2 dimensional area integrand in $R^{3}$ and consider the problem of finding a function $f: R^{2} \cap\left\{(x, y): x^{2}+y^{2} \leqq 1\right\} \rightarrow R$ such that $f(x, y)=0$ for $x^{2}+y^{2}=1$ and whose graph $\left\{(x, y, z): x^{2}+y^{2} \leqq 1\right.$ and $z=f(x, y)\}$ has the smallest area among all such functions. In particular, we are asking that $\int$ satisfy the minimal surface equation. The unique solution is, of course, $f \equiv 0$. Now let $h: R^{3} \rightarrow R^{3}$ be a class $\infty$ diffeomorphism which leaves $\left\{(x, y, z): x^{2}+y^{2}=1\right.$ and $\left.z=0\right\}$ fixed, carries $\{(x, y, z):-1 / 2 \leqq x \leqq 1 / 2, y=z=0\}$ isometrically onto $\{(x, y, z):-1 / 2 \leqq z \leqq 1 / 2, x=y=0\}$, and carries $\left\{(x, y, z): x^{2}+y^{2}\right.$ $\leqq 1, z=0\} \sim\{(x, y, z):-1 / 2 \leqq x \leqq 1 / 2, y=z=0\}$ onto the graph of a class $\infty$ function $g:\left\{(x, y): 0<x^{2}+y^{2} \leqq 1\right\} \rightarrow R$ (see Figure 10).

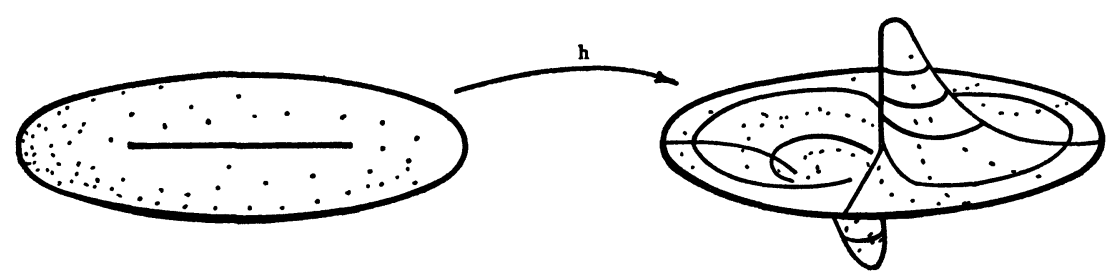

FIGURE 10.

The function $g$ is clearly the unique natural solution to the Euler partial differential equation associated with the variational problem for the integrand $h \#$. This partial differential equation is elliptic and of class $\infty . g$, of course, is not continuous at $(0,0)$.

7. Minimal integral currents. As has been indicated there are a number of different natural formulations of calculus of variations problems in the measure theoretic setting. For the purpose of the theorem in $\$ 5$, we considered surfaces as compact rectifiable sets without orientations and without multiplicities. The singularities which result (or do not result) in solutions to these problems are very much a function of the manner in which the problem is formulated. It is useful to give examples. First consider the problem of finding 1 dimensional surfaces of least length in $R^{2}$ corresponding to homology boundary conditions with integer coefficients. Suppose the boundary $B$ consists of four points spaced as in Figure 11-1. In this figure we have specified + and - orientations for these points. We can regard these four points with their associated +'s and -'s as a nonbounding cycle representing a homology class $\sigma \in H_{0}(B ; Z)$. The 
1 dimensional surface $S$ of least $H_{1}$ measure for which $i_{*}(\sigma)=0$ is indicated in Figure 11-2, where $i_{*}: H_{0}(B ; Z) \rightarrow H_{0}(B \cup S ; Z)$ is induced by the inclusion $i: B \rightarrow B \cup S$. One checks that $H_{1}(S)=4+10 \cdot 3^{-1 / 2}$. One can also regard the four oriented points of $B$ as a 0 dimensional integral current $B^{*} \in Z_{v}(Z)$. The 1 dimensional integral current $S^{*} \in Z_{1}(Z)$ having least mass $M$ among all such currents $T^{*}$ with
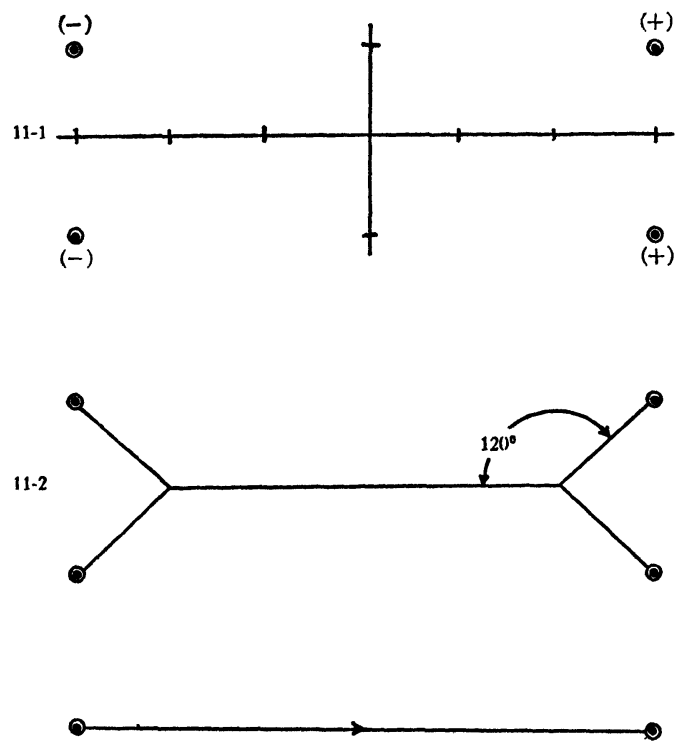

$11-3$

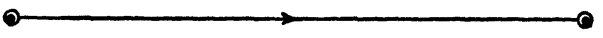

Figure 11.

$\partial T^{*}=B^{*}$ is indicated in Figure 11-3. In particular, $M\left(S^{*}\right)$ $=12>H_{1}(S)$. The first formulation of the problem above in which surfaces are compact rectifiable sets gives the smaller total length of its solution surface, and, in general, this formulation gives the least value of the integral of an elliptic integrand for variational problems in all dimensions and codimensions when the boundary conditions are homological. However, the solution surface $S$ has two singularities of codimension 1, i.e. the two points where three line segments meet, while the integral current solution has no interior singularities. 
If we consider the analogous problem for 2 dimensional minimal surfaces in $R^{3}$ having a boundary $B$ consisting of two appropriately spaced parallel circles with the orientations indicated in Figure 12-1, the 2 dimensional surface of least $\boldsymbol{H}_{2}$ measure spanning the homology class in $H_{1}(B ; Z)$ represented by $B$ probably looks like the surface in Figure 12-2 which has a circle for its interior singular set-this surface, incidentally, is in the shape of the soap film which most
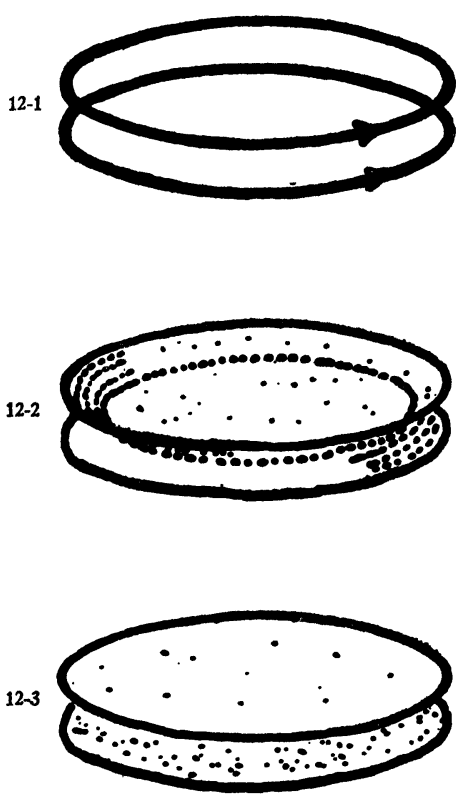

Figure 12.

commonly forms on such a boundary. The integral current of least mass whose boundary is the 1 dimensional integral current represented by $B$ consists of two parallel disks and, in particular, has no interior singularities (Figure 12-3).

One often thinks of a piece of a catenoid as a surface of least area associated with the boundary $B$. This is the solution surface if one orients the lower circle in $B$ in the opposite direction. For $B$ so oriented, the integral current consisting of two suitably oriented parallel disks has boundary $B$ and is locally of least mass. From topological considerations, one can show from the existence of two distinct minimal surfaces, each of least area locally, the existence of a third, gen- 
erally "unstable," minimal surface having the boundary $B$ and lying inside the piece of catenoid which has least area.

As the examples above suggest, one sometimes can formulate Plateau's problem in such a way as to avoid interior singularities in the solutions. The following theorem (with an obvious extension to real analytic manifolds) represents what is presently known.

Theorem [R1], [FF, 8.13], [FL3, 1], [FL2, 4], [A4], [S,6, 2.1], [AL]. Let $2 \leqq n \leqq 7$ be an integer and $T \in Z_{n-2}(Z)$ in $R^{n}$ [resp. $T$ $\in Z_{n-2}\left(Z_{2}\right)$ in $\left.R^{n}\right]$ with $\partial T=0$. Then there exists $Q \in Z_{n-1}(Z)$ [resp. $Q$ $\in Z_{n-1}\left(Z_{2}\right)$ ] of least mass among all $Z$ chains [resp. $Z_{2}$ chains] having boundary $T$. For any such $Q$ of least mass, $\operatorname{spt}(Q) \sim \operatorname{spt}(T)$ is a real analytic oriented [resp. unoriented] submanifold of $R^{n}$ of dimension $n-1$ having zero mean curvature at every point. If $k \geqq 3$ and $T$ is a class $k$ \{resp.real analytic $\}$ oriented [resp. unoriented] submanifold of dimension $n-2$ lying on the boundary of a uniformly convex subset of $R^{n}$, then $\operatorname{spt}(Q)$ is a class $k-1$ \{resp. real analytic\} manifold with boundary.

W. H. Fleming and E. De Giorgi have shown that the above interior regularity result implies the following extension of Bernstein's Theorem.

Theorem [FL2, 5], [DG, 2], [A4], [S, 6.2.2]. Let $3 \leqq n \leqq 8$ and $f: R^{n-1} \rightarrow R$ be a class 3 function satisfying the minimal surface equation. Then the graph of $f, R^{n} \cap\left\{x: x^{n}=f\left(x^{1}, \cdots, x^{n-1}\right)\right\}$, is a hyperplane in $R^{n}$.

\section{REFERENCES}

AL. W. K. Allard, On boundary regularity for Plateau's problem, Bull. Amer. Math. Soc. 75 (1969).

A1. F. J. Almgren, Jr., The homotopy groups of the integral cycle groups, Topology 1 (1962), 257-299.

A2. - The theory of varifolds. A variational calculus in the large for the $k$ dimensional area integrand (multilithed notes), Princeton, 1964.

A3. - Plateau's Problem. An Invitation to Varifold Geometry, W. A. Benjamin, Inc., New York, 1966.

A4. - Some interior regularity theorems for minimal surfaces and an extension of Bernstein's theorem, Ann. of Math. (2) 84 (1966), 277-292.

A5. - Existence and regularity almost everywhere of solutions to elliptic variational problems among surfaces of varying topological type and singularity structure, Ann. of Math. (2) 87 (1968), 321-391.

B. J. E. Brothers, Integral geometry in homogeneous spaces, Trans. Amer. Math. Soc. 124 (1966), 480-517.

D. J. Douglas, Solution of the problem of Plateau, Trans. Amer. Math. Soc. 33 (1931), 263-321. 
DG1. E. De Giorgi, Frontiere orientate di misura minima, Sem. Mat. Scuola Norm. Sup. Pisa, 1960/61, pp. 1-56.

DG2. - Una estensione del teorema di Bernstein, Ann. Scuola Norm. Sup. Pisa, (3) 19 (1965), 79-85.

F1. H Federer, The $(\phi, k)$ rectifiable subsets of $n$ space, Trans. Amer. Math. Soc. 62 (1947), 114-192.

F2. - - Curvature measures, Trans. Amer. Math. Soc. 93 (1959), 418-491.

F3. - Currents and area, Trans. Amer. Math. Soc. 98 (1961), 204-233.

F4. - Some theorems on integral currents, Trans. Amer. Math. Soc. 117 (1965), 43-67.

F5. - Geometric Measure Theory, Springer-Verlag, New York, 1969.

FF. H. Federer and W. H. Fleming, Normal and integral currents, Ann. of Math.

(2) 72 (1960), 458-520.

FL1. W. H. Fleming, An example in the problem of least area, Proc. Amer. Math. Soc. 7 (1956), 1065-1074.

FL2. - On the oriented Plateau Problem, Rend. Circ. Mat. Palermo (2) 11 (1962), 69-90.

FL3. - Flat chains over a finite coefficient group, Trans. Amer. Math. Soc. 121 (1966), 160-186.

GM. E. Giusti and M. Miranda, Sulla regolaritd delle soluzioni deboli di una classe di sistemi ellittici quasi-lineari, Arch. Rational Mech. Anal. (to appear).

M1. C. B. Morrey, Multiple Integrals in the Calculus of Variations, SpringerVerlag, New York, 1960.

M2. - Partial regularity results for non-linear elliptic systems, J. Math. Mech. 17 (1968), 649-670.

PS. R. S. Palais and S. Smale, $A$ generalized Morse theory, Bull. Amer. Math. Soc. 70 (1964), 165-172.

R1. E. R. Reifenberg, Solution of the Plateau problem for m-dimensional surfaces of varying topological type, Acta Math. 104 (1960), 1-92.

R2. - An epiperimetric inequalily related to the analyticity of minimal surfaces, Ann. of Math. (2) 80 (1964), 1-14.

R3. - On the analyticity of minimal surfaces, Ann. of Math. (2) 80 (1964), 15-21.

S. J. Simons, Minimal varieties in riemannian manifolds, Ann. of Math. (2) 88 (1968), 62-105.

T. R. Thom, Quelques proprietes globales des variêtes differentiables, Comment. Math. Helv. 28 (1954), 17-86.

Princeton University. Princeton, New Jersey 08540 